\title{
Multiparametric Magnetic Resonance Imaging of the Prostate: An Update
}

\author{
Savinay Kapur ${ }^{1}$ Chandan J. Das ${ }^{1}$ Sanjay Sharma ${ }^{1}$ \\ ${ }^{1}$ Department of Radiodiagnosis, All India Institute of Medical \\ Sciences, Ansari Nagar, New Delhi, India
}

Ann Natl Acad Med Sci (India) 2019;55:74-83

\begin{abstract}
Address for correspondence Chandan J. Das, MD, DNB, MAMS, FICR, FRCP, Department of Radiodiagnosis, All India Institute of Medical Sciences, Sri Aurobindo Marg, Ansari Nagar, New Delhi 110029, India (e-mail: docchandan17@gmail.com).
\end{abstract}

\begin{abstract}
Keywords

- multiparametric MRI

- prostate cancer

- PIRADS

Multiparametric magnetic resonance imaging (mp-MRI) has emerged as an important tool for the detection and characterization of prostatic lesions. It now plays a quintessential role in the surveillance, diagnosis, and staging of prostate cancer (PCa), as well as for the detection of local recurrence. As reliance on serum prostate-specific antigen has declined in the recent times, mp-MRI has emerged as the go-to tool for urologists all over the world. Hence, for the clinician, it has become necessary to be well versed with the technique, image interpretation, and fallacies of mp-MRI. Since mp-MRI has the advantage of better contrast resolution, combining PSMA PET (prostate-specific membrane antigen-positron emission tomography) with MRI could provide additional functional information. However, due to the absence of enough evidence supporting its routine use, mp-MRI still has the unsurpassed role in the initial diagnosis and local staging of PCa.
\end{abstract}

\section{Introduction}

Multiparametric magnetic resonance imaging (mp-MRI) has emerged as an important tool for the detection and characterization of prostatic lesions. It now plays a quintessential role in the surveillance, diagnosis, and staging of prostate cancer (PCa), as well as for the detection of local recurrence. As reliance on serum prostate-specific antigen (PSA) has declined in the recent times, mp-MRI (T2-weighted [T2W] images, diffusion-weighted imaging [DWI], dynamic contrast-enhanced [DCE] MRI, and magnetic resonance [MR] spectroscopy [MRS]) has emerged as the go-to tool for urologists all over the world. Hence as radiologists, it has become necessary to be well versed with the technique, image interpretation, and fallacies of mp-MRI. This review is aimed at providing a brief overview of prostatic MRI, its indications, and basics of sequences used, with emphasis on recent updates in MRI of the prostate.

PCa is one of the most commonly diagnosed malignancies in men. The age-adjusted incidence rate in India is 37 per 100,000 men per year. ${ }^{1}$ Difficulty in accurate staging and prediction of disease progression has made the management of PCa a complex issue. Traditionally, urologists have relied on digital rectal examination (DRE), PSA levels, and transrectal ultrasound (TRUS) with sextant biopsy for the diagnosis of PCa. ${ }^{2}$ MRI, in the present scenario, has become indispensable for not only staging of prostatic cancer but also for its surveillance, detection, and follow-up. Radiologists can be easily intimidated by prostate MRI examinations. Knowledge of glandular anatomy, the use and interpretation of various MRI sequences, and, finally, the reporting of correct findings is challenging for an amateur reader. Although the technical details of imaging protocols are beyond the scope of this paper, the standard imaging sequences that constitute multiparametric prostate imaging will be discussed along with their utility. The aim of this review is to discuss, in brief, the fundamental anatomy of the prostate and the techniques of mp-MRI and to provide a guide for the interpretation and reporting of mp-MRI scans.

\section{Normal Anatomy of the Prostate}

The prostate is grossly divided into three anatomical regions: the base, the midgland, and the apex. The base forms the 
broad and superior most limit of the prostate and is located below the urinary bladder neck. The narrower apex rests below on the pelvic floor. Each anatomical region consists of a left side and a right side, thus dividing the prostate into six parts or sextants. Biopsy samples are obtained from each sextant, and this organization helps in clinic-radiologicpathological correlation.

The anterior fibromuscular stroma, the transition zone (TZ), the central zone (CZ), and the outer peripheral zone (PZ) form the four histological zones. ${ }^{3}$ The nonglandular anterior fibromuscular stroma may contribute to up to one-third of the prostatic mass. It may be replaced by glandular tissue in adenomatous enlargement of the prostate. However, it is rarely invaded by carcinoma. The TZ surrounds the urethra proximal to the verumontanum and contains $5 \%$ of the glandular tissue. It is the TZ that accounts for the increase in size of the prostate in benign prostatic hyperplasia (BPH) by increasing the percentage of the gland volume. It is estimated that only 20 to $30 \%$ of PCas originate in this zone. The CZ expands in a cone shape around the ejaculatory ducts to the base of the bladder and contains approximately $20 \%$ of the glandular tissue. Only 1 to $5 \%$ of adenocarcinomas arise in the $\mathrm{CZ}$, although it may be infiltrated by tumors arising from the PZ. The PZ forms the bulk of the glandular tissue (70-80\%), and 70 to $75 \%$ PCas arise from this zone. ${ }^{4}$

On T1-weighted (T1W) images, the entire prostate gland appears uniform with intermediate signal intensity (SI). Hence, the zonal anatomy of the prostate cannot be made out on T1W images. ${ }^{2}$ The location and differences in SI on T2W images often help in distinguishing $\mathrm{TZ}$ from the $\mathrm{CZ}$ on MR images ( - Fig. 1). The anterior fibromuscular stroma is seen as dark on both $\mathrm{T} 1$ and $\mathrm{T} 2 \mathrm{~W}$ images. The PZ, on the other hand, is bright on T2W images, having SI comparable to the adjacent periprostatic fat. It is bound by a T2 hypointense capsule. Both CZ and TZ tend to be of lower T2 SI than the PZ possibly because of more compact smooth muscle and lesser glandular elements. Age-related expansion of TZ seen in BPH, however, may result in compression and displacement of the $\mathrm{CZ} .^{5}$

The "prostate capsule," which is a thin, dark rim surrounding the prostate gland on $\mathrm{T} 2 \mathrm{~W}$ images, serves as an important landmark for the assessment of extraprostatic extension (EPE) of cancer. It is incomplete anteriorly and apically and contains an outer band of concentric fibromuscular tissue that is inseparable from the prostatic stroma. Thus, the prostate lacks a true capsule. The prostatic pseudocapsule (sometimes called "surgical capsule" when seen at enucleation of an adenoma) on T2W MRI, is a thin, dark rim at the interface of the TZ with the PZ, which appears so due to compressed prostate tissue.

The proximal urethra cannot be visualized on MR unless there is a Foley catheter in situ or a transurethral resection has been performed. The verumontanum appears as a T2 hyperintense structure in the midline posterior to the urethra. The vas deferens and seminal vesicles are best seen on axial and coronal images. ${ }^{2}$

The neurovascular bundles (NVBs) to the prostate are formed by the sympathetic and parasympathetic nerves that supply the corpora cavernosa and the closely associated arterial branches from the inferior vesicle artery. Together, these course posterolateral to the prostate bilaterally, at 5 and 7 o'clock positions. Small nerve branches that surround the prostate periphery and penetrate through the capsule at the apex and base form a potential route for the EPE of cancer. The NVBs are best seen on axial images. ${ }^{2}$

\section{Indications for Prostate MRI}

MRI has been used for the assessment of the prostate since the 1980s, when it was used largely for the staging of biopsy-proven prostate carcinoma. The locoregional spread of the disease was evaluated based on T1W and T2W pulse sequences, which helped describe the morphology of the prostate. ${ }^{6}$

In the recent times, however, mp-MRI has been developed to significantly improve the sensitivity and specificity of MRI for carcinoma prostate. mp-MRI integrates anatomical, functional, and physiological assessments using T2W images, DWI, dynamic contrast enhancement, and MR proton spectroscopy.

Consequently, prostate MRI is now being used for the detection of tumors and their localization and characterization and for the prognostication as well as assessment of suspected recurrent disease. It may even be employed for taking image-guided biopsies and targeted therapy. ${ }^{7.8}$

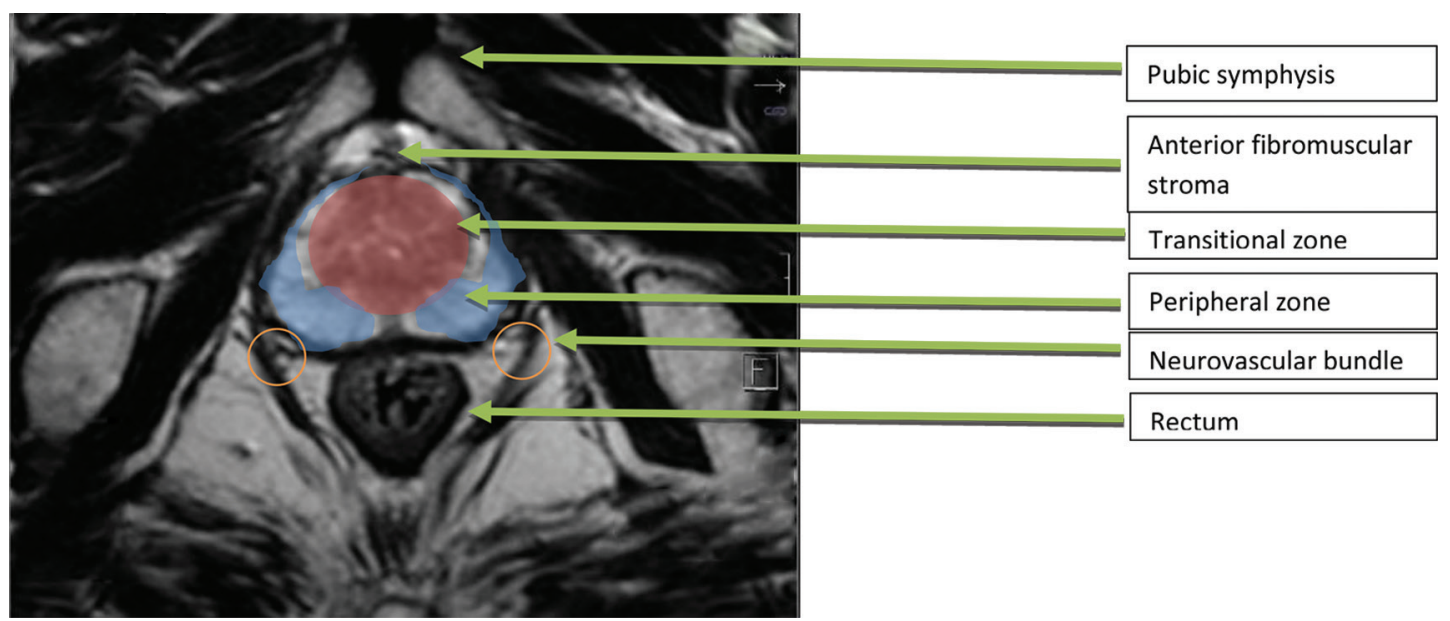

Fig. 1 Normal anatomy of the prostate. 
The extensive use of mp-MRI, however, faced several impediments. The European Society of Urogenital Radiology (ESUR) drafted guidelines along with a scoring system to standardize its use. The scoring system is known as Prostate Imaging Reporting and Data System version 1 (PIRADS v1). ${ }^{8}$ This effort generated interest in the use of mp-MRI for the prostate. Moreover, it provided validation and consistency to imaging findings, which encouraged widespread use. Nonetheless, the system had limitations in several clinical scenarios. To resolve the deficiencies, the American College of Radiology, the ESUR, and the AdMeTech Foundation improved upon PIRADS v1 in the form of PIRADS v2 (2015). PIRADS v2 is designed to "promote global standardization and diminish variation in the acquisition, interpretation, and reporting of prostate mp-MRI examinations." ${ }^{6}$ It provides minimum acceptable technical parameters for performing a prostate mp-MRI and standardizing the terminology and content of radiology reports. The basic aim of PIRADS is to reduce the variability in imaging interpretation and to enhance communication between radiologists and the referring clinicians.

Despite the updated version, the limitation of use of PIRADS for the treatment of naïve prostate glands remains. Thus, its use for the detection of suspected recurrence or progression is not validated.

\section{Clinical Considerations and Technical Specifications}

\section{Clinical Considerations}

Timing of MRI following Prostate Biopsy

Hemorrhage and postbiopsy changes can possibly confound MRI interpretation. Hemorrhage appears as bright on the T1W images and is generally seen in the PZ or in the seminal vesicles following a TRUS-guided biopsy. Two general approaches can be followed to avoid this potential pitfall. Although hemorrhagic changes can persist for months following a biopsy, they generally resolve within 6 weeks. Hence, ideally, an MRI should be performed at least 6 weeks after a biopsy. ${ }^{6}$ However, since waiting for 6 weeks may not always be feasible, an initial $\mathrm{T} 1$ sequence may be performed as a screening. If there is evidence of hemorrhage, the MRI may be postponed after discussion with the urologist. If postponing the MRI is not an option, it must be remembered that the possibility of finding a malignant lesion at the site of postbiopsy hemorrhage is low. In such a situation, it is imperative to rule out malignancy at locations other than those showing hemorrhagic change. ${ }^{9}$

\section{Patient Preparation}

The use of enema for rectal emptying is controversial. The presence of air/stool in the rectum can cause distortion of MR signal and compromise DWI quality. However, enemas also promote peristalsis, resulting in increased motion-related artifact. Antispasmodics such as glucagon and scopolamine can reduce these motion-related artifacts; however, their use must be weighed against the cost and possible adverse effects associated with their use.
Though there are no guidelines at present, evacuation of the rectum by the patient prior to the MRI generally obviates the need for enema.

If air is seen on the initial MR images, it may be worthwhile to perform the MRI with the patient in the prone position or to use a suction catheter for evacuation. ${ }^{6}$

\section{Patient Information}

As is repeatedly emphasized, knowledge of the patient's history and clinical findings is always beneficial while interpreting images. It is important to record the patient's recent serum PSA level and PSA history. If a biopsy has been performed, the results of the biopsy including the number and location of positive cores and corresponding Gleason scores are of immense value to the reporting radiologist.

\section{Technical Specifications of Equipment}

Three sequences are essential for a prostate mp-MRI according to the PIRADS v2. These include T2W, DWI, and DCE. The role of MRS is controversial, but we will still cover it in this review. Apart from being used for the confirmation and localization of clinically significant malignancy, mp-MRI also has the potential of being used as a screening modality. However, cost and time are the two limiting factors. As a corollary, the supervising radiologist should be vigilant about not including unnecessary sequences as it not only affects the patient compliance and acceptance but also reduces the machine throughput.

The field of view should be reduced to include the relevant structures only. However, at least one pulse sequence should include area up to the aortic bifurcation. This allows for the evaluation of pelvic nodes. ${ }^{6}$

\section{Field Strength: 3T or 1.5T}

With increasing field strength, there is a linear increase in the signal-to-noise ratio (SNR). Hence, employing a 3T magnetic field instead of a $1.5 \mathrm{~T}$ one means that the spatial ratio and temporal resolution both can be increased. The downside is that increasing the field strength also increases power deposition, artifacts related to susceptibility, and signal heterogeneity. However, the advantages of 3T scanners over 1.5T significantly outweigh the disadvantages. PIRADS v2 recommends the use of $3 \mathrm{~T}$ for prostate MRI. The only indication for using a $1.5 \mathrm{~T}$ scanner is when a patient has an implant that is either compatible only with $1.5 \mathrm{~T}$ or is in a location that could result in significant artifacts during imaging, such as a hip prosthesis. ${ }^{6}$

\section{Endorectal Coils or Surface Coils}

Endorectal coils (ERCs) offer the advantage of increased SNR with the obvious disadvantage of patient discomfort and poor acceptance apart from possible problems such as deformation of the gland and artifacts. The increased spatial resolution is especially advantageous while using lower SNR sequences such as DWI and high temporal resolution DCE. It also scores over external phased array radiofrequency (RF) coils in obese patients. ${ }^{6}$

At 1.5T, ERC was indispensable for obtaining high-resolution diagnostic quality imaging. However, at 3T, surface coils 
can provide equally good quality imaging. Some of the $1.5 \mathrm{~T}$ scanners that employ a relatively high number of external phased array coil elements and RF channels (e.g., 16 or more) may be capable of achieving adequate SNR in many patients without an ERC as multiple factors including receiver bandwidth, coil design, and efficiency of the RF chain. ${ }^{6}$

PIRADS v2 recommends optimization of protocols and coils to obtain the best possible image quality, taking into account the cost, availability, and patient preference. No specific recommendation has been made regarding the use of ERC/surface coils.

\section{Computer-Aided Evaluation Technology}

Computer-aided evaluation (CAE) can enhance lesion detection and discrimination performance of amateur radiologists who have little or no experience in reading prostate MRIs. However, CAE using specialized software or a dedicated workstation is not required for prostate mp-MRI interpretation. ${ }^{6}$

\section{Multiparametric MRI}

\section{T1- and T2-Weighted Images}

T1W images are obtained to detect the presence of hemorrhage within the prostate or seminal vesicles and to outline the anatomy of the region.

T2W imaging is the best imaging sequence to delineate the prostatic anatomy, for the detection and categorization of lesions, and for the EPE and nodal assessment. ${ }^{10}$

T2W imaging is the dominant sequence to be assessed in the TZ. Features of malignancy in the TZ are noncircumscribed, ill-defined, homogeneously hypointense lesions that have been variously described as erased charcoal or smudgy fingerprint appearance. Other features of malignancy include spiculated margins, lenticular shape, absence of a complete hypointense capsule, extension of the lesion into adjacent structures such as the urethral sphincter, and anterior fibromuscular stroma. Though no single feature is not diagnostic of malignancy, the likelihood of malignancy increases with the presence of more number of features.

TZ is composed of variable amounts of glandular (T2 hyperintense) and stromal (T2 hypointense) elements, and this makes the overall signal of the TZ heterogeneous. Hence, detection of malignancy is especially challenging. Areas where benign stromal elements predominate can mimic clinically significant malignancy.

In the PZ, clinically significant cancer tends to occur as a well-defined, round, focal hypointense lesion. However, mimics such as hemorrhage, prostatitis, glandular atrophy, and benign hyperplasia can mimic malignancy.

A relatively specific sign of malignancy is extension of the lesion across anatomical boundaries. T2W images can help discern extension of the lesion within the gland (across regional parts of the prostate) as well as into the seminal vesicles/adjacent fat and NVB.

Three-dimensional (3D) axial acquisition can help improve the spatial resolution with isotropic voxels. However, the soft tissue contrast and in-plane resolution tend to be inferior to routine $2 \mathrm{D}$ acquisition. At our institute, we do not routinely acquire 3D datasets.

\section{Diffusion-Weighted Imaging}

One sequence that has had a significant role in the increased use and popularity of mp-MRI is DWI. It is based on the principle of free, random motion of water molecules. DWI includes an apparent diffusion coefficient (ADC) map and high $b$-value images (-Table $\mathbf{1}$ ).

The ADC map is a representation of the ADC values of each voxel in the image. It uses two or more $b$-values and a monoexponential model of signal decay with increasing $b$-values to calculate ADC values. Clinically significant cancers have restricted diffusion as compared with normal tissue and appear hyperintense on high $b$-value images and hypointense on ADC maps. Quantitatively, ADC values correlate inversely with the tumor grade, and though attempts have been made to provide cutoffs for ADC values for predicting malignant lesions, there is considerable overlap between benign prostatic nodules and low- and high-grade malignant lesions. Though a threshold of 750 to $900 \mathrm{um}^{2} /$ second has been suggested to differentiate benign from malignant prostatic lesions in the PZ, qualitative visual assessment remains the most commonly employed and used technique.

DWI of the prostate is unique. It uses high $b$-value images $\left(>1,400\right.$ seconds $\left./ \mathrm{m}^{2}\right)$ that are not routinely used in imaging of other body parts. DWI is highly sensitive to motion

Table 1 PIRADS v2 assessment for T2W image

\begin{tabular}{|c|c|}
\hline \multicolumn{2}{|c|}{ Peripheral zone } \\
\hline Score & Lesion characteristics \\
\hline 1 & Uniform hyperintense signal intensity (normal) \\
\hline 2 & $\begin{array}{l}\text { Linear or wedge-shaped hypointensity or diffuse } \\
\text { mild hypointensity, usually indistinct margin }\end{array}$ \\
\hline 3 & $\begin{array}{l}\text { Heterogeneous signal intensity or noncircum- } \\
\text { scribed, rounded, moderate hypointensity } \\
\text { Includes others that do not qualify as } 2,4 \text {, or } 5\end{array}$ \\
\hline 4 & $\begin{array}{l}\text { Circumscribed, homogenous moderate hy- } \\
\text { pointense focus/mass confined to the prostate and } \\
<1.5 \mathrm{~cm} \text { in greatest dimension }\end{array}$ \\
\hline 5 & $\begin{array}{l}\text { Same as } 4 \text { but } \geq 1.5 \mathrm{~cm} \text { in greatest dimension or } \\
\text { definite extraprostatic extension/invasive behavior }\end{array}$ \\
\hline \multicolumn{2}{|c|}{ Transition zone } \\
\hline Score & Lesion characteristics \\
\hline 1 & $\begin{array}{l}\text { Homogeneous intermediate signal intensity } \\
\text { (normal) }\end{array}$ \\
\hline 2 & $\begin{array}{l}\text { Circumscribed hypointense or heterogeneous } \\
\text { encapsulated nodule(s) (BPH) }\end{array}$ \\
\hline 3 & $\begin{array}{l}\text { Heterogeneous signal intensity with obscured mar- } \\
\text { gins, includes others that do not qualify as } 2,4 \text {, or } 5\end{array}$ \\
\hline 4 & $\begin{array}{l}\text { Lenticular or noncircumscribed, homogeneous, } \\
\text { moderately hypointense, and }<1.5 \mathrm{~cm} \text { in greatest } \\
\text { dimension }\end{array}$ \\
\hline 5 & $\begin{array}{l}\text { Same as } 4 \text { but } \geq 1.5 \mathrm{~cm} \text { in greatest dimension or } \\
\text { definite extraprostatic extension/invasive behavior }\end{array}$ \\
\hline
\end{tabular}

Abbreviations: BPH, benign prostatic hyperplasia; PIRADS, Prostate Imaging Reporting and Data System; T2W, T2-weighted. 
and is a time-consuming imaging technique. As the $b$-value increases, the spatial resolution decreases. At such high $b$-values though, only the areas with restricted diffusion show signal and appear bright. This increases the conspicuity of lesions especially in the subcapsular location at the base and apex of the gland as well as adjacent to the anterior fibromuscular stroma.

High $b$-value images can be obtained in two ways: by directly acquiring a high $b$-value sequence (time consuming) or by extrapolating data acquired at lower $b$-values to create the ADC maps. The extrapolation time has two advantages: less time and less artifact. This is achieved by avoiding the longer echo times required to accommodate the strong gradient pulses required for higher $b$-value acquisitions.

If only two $b$-values can be acquired, the lower $b$-value should be set at 50 to 100 seconds $/ \mathrm{mm}^{2}$ and the higher $b$-value should be 800 to 1,000 seconds $/ \mathrm{mm}^{2}$. High $b$-value images $\left(>1,400\right.$ seconds $/ \mathrm{mm}^{2}$ ) can be extrapolated. For more accurate ADC calculation and extrapolation of high $b$-value images, additional $b$-values may be obtained between 100 and 1,000 (-Table 2).

\section{Dynamic Contrast-Enhanced MRI}

DCE MRI involves acquisition of scans just before, during, and after contrast injection to study the enhancement characteristics of lesions. Rapid T1W gradient-echo scans are obtained every few seconds with injection of a bolus of gadolinium-based contrast. PCas show early enhancement compared with normal tissue with washout. However, prostate malignancy shows variable and heterogeneous contrast kinetics. Some tumors may show early washout, whereas others may show contrast retention. Enhancement is neither a sign of malignancy nor does the absence of enhancement exclude a diagnosis of prostate malignancy.

The importance of DCE lies in the fact that it can pick up small lesions, which may show focal early enhancement. Any small lesion showing focal early enhancement should be carefully looked at in the corresponding T2W images and DWI. However, its role in the characterization of lesions is limited and subordinate to T2W images and DWI.

Positive early enhancement can be compared with the adjacent normal prostatic tissue. It usually occurs within 10 seconds of appearance of contrast in the femoral artery.

Table 2 PIRADS v2 assessment for DWI

\begin{tabular}{|l|l|}
\hline Score & Lesion characteristics \\
\hline 1 & No abnormality on ADC and high $b$-value DWI \\
\hline 2 & Indistinct hypointense on ADC \\
\hline 3 & $\begin{array}{l}\text { Focal mildly/moderately hypointense on ADC and } \\
\text { isointense/mildly hyperintense on high } b \text {-value DWI }\end{array}$ \\
\hline 4 & $\begin{array}{l}\text { Focal markedly hypointense on ADC and marked- } \\
\text { ly hyperintense on high } b \text {-value DWI, }<1.5 \mathrm{~cm} \text { in } \\
\text { greatest dimension }\end{array}$ \\
\hline 5 & $\begin{array}{l}\text { Same as } 4 \text { but } \geq 1.5 \mathrm{~cm} \text { in greatest dimension or } \\
\text { definite extraprostatic extension/invasive behavior }\end{array}$ \\
\hline
\end{tabular}

Abbreviations: ADC, apparent diffusion coefficient; DWI, diffusionweighted imaging; PIRADS V2, Prostate Imaging Reporting and Data System Version 2.
Contrast enhancement for a lesion is reported as either positive or negative. Positive DCE refers to focal enhancement of a lesion that is earlier or simultaneous with enhancement of normal prostatic tissue and corroborating with findings on T2W images and/or DWI. Negative DCE is defined by the absence of early enhancement or diffuse enhancement not corresponding to a focal finding on T2 and/or DWI or focal enhancement corresponding to a lesion demonstrating features of BPH on T2W images.

Diffuse enhancement in the prostate is usually associated with prostatitis. Infiltrating malignancy may also show diffuse enhancement, but these lesions show corresponding signal changes on T2W images and DWI as well. At times, histologically sparse PCas are intermixed with benign prostatic tissues. These tumors tend to be occult on T2W images and DWI and may be apparent only on DCE. However, these are usually lower grade malignancies, and the enhancement may be due to concurrent prostatitis.

The easiest and the most commonly used technique for interpretation of DCE is direct visual assessment. The various DCE time points at each slice location are assessed either by manual scrolling or using cine mode. It is recommended to use fat suppression or subtraction technique to improve lesion pickup. Using a parametric map which color-codes enhancement features within a voxel (such as slope and peak) can also assist image interpretation. However, any suspicious finding on a parametric map or subtracted images should be confirmed on the source images.

Another technique used for the interpretation of dynamic contrast enhancement is plotting the SI of the lesion versus time: curve typing. However, given the heterogeneity in enhancement characteristics of PCa, this has not proven to be too useful. Perfusion parameters like $K^{\text {trans }}$ (wash-in) and $K_{\text {ep }}$ (washout) have been used to assist in diagnosis. As of now, not enough published literature is available to recommend the use of perfusion parameters for the diagnosis of prostate malignancy (-Table 3 ).

\section{PIRADS V2 Reporting}

PIRADS, like the already established BI-RADS, aims at standardizing assessment and reporting of mp-MRI for PCa. It uses a 5-point scale to categorize the probability of malignancy. The aim of PIRADS is to pick up all clinically significant malignancies while reducing unnecessary biopsies.

Clinically significant $\mathrm{PC}$ a has been defined as pathologically proven PCa with Gleason score $\geq 7$ (including $3+4$ with prominent but not predominant Gleason 4 component), volume $\geq 0.5 \mathrm{~mL}$, and/or EPE.

The PIRADS assessment is based on a combination of T2W, DWI, and DCE findings, with the relative importance of these sequences differing according to the zone of the gland (-Figs. 2-6).

Biopsy should be considered for all PIRADS 4 and 5 lesions, whereas PIRADS 1 categorizes no-touch lesions. Lesions that are PIRADS 2 or 3 require correlation with PSA/DRE and other clinical details for their management. Depending on the clinical and laboratory findings as well as the local preferences 
Table 3 Magnetic resonance imaging sequence parameters

\begin{tabular}{|l|l|l|l|}
\hline \multicolumn{1}{|c|}{ T2W } & \multicolumn{1}{|c|}{ DWI } & \multicolumn{1}{c|}{ DCE } \\
\hline Sequence & TSE/FSE & $\begin{array}{l}\text { Free-breathing spin-echo EPI with spectral } \\
\text { fat saturation (TE } \leq 90 \mathrm{~ms}, \mathrm{TR} \geq 3,000 \mathrm{~ms})\end{array}$ & $\begin{array}{l}\text { 2D/3D T1 GRE sequence } \\
\text { TR }<100 \mathrm{~ms}, \mathrm{TE}<5 \mathrm{~ms}\end{array}$ \\
\hline $\begin{array}{l}\text { Plane of } \\
\text { imaging }\end{array}$ & Axial, coronal, sagittal & Axial & Axial \\
\hline $\begin{array}{l}\text { Slice } \\
\text { thickness }\end{array}$ & $3 \mathrm{~mm}$ (no gap) & $\leq 4 \mathrm{~mm}$ & $3 \mathrm{~mm}$ (no gap) \\
\hline FOV & $\begin{array}{l}12-20 \mathrm{~cm} \text { (to encompass the en- } \\
\text { tire prostate gland and seminal } \\
\text { vesicles) }\end{array}$ & $16-22 \mathrm{~cm}$ & $\begin{array}{l}\text { Encompass the entire prostate } \\
\text { gland and seminal vesicles }\end{array}$ \\
\hline $\begin{array}{l}\text { In-plane } \\
\text { dimension }\end{array}$ & $\begin{array}{l}\leq 0.7 \mathrm{~mm} \text { (phase) } \times \leq 0.4 \mathrm{~mm} \\
\text { (frequency) }\end{array}$ & $\leq 2.5 \mathrm{~mm}$ (phase and frequency) & $\leq 2 \times \leq 2$ mm \\
\hline
\end{tabular}

Abbreviations: DCE, dynamic contrast enhancement; DWI, diffusion-weighted imaging; EPI, echo planar imaging; FOV, field of view; FSE, fast spin echo; GRE, gradient echo; T2W, T2-weighted; TE, echo time; TR, repetition time; TSE, turbo spin echo.

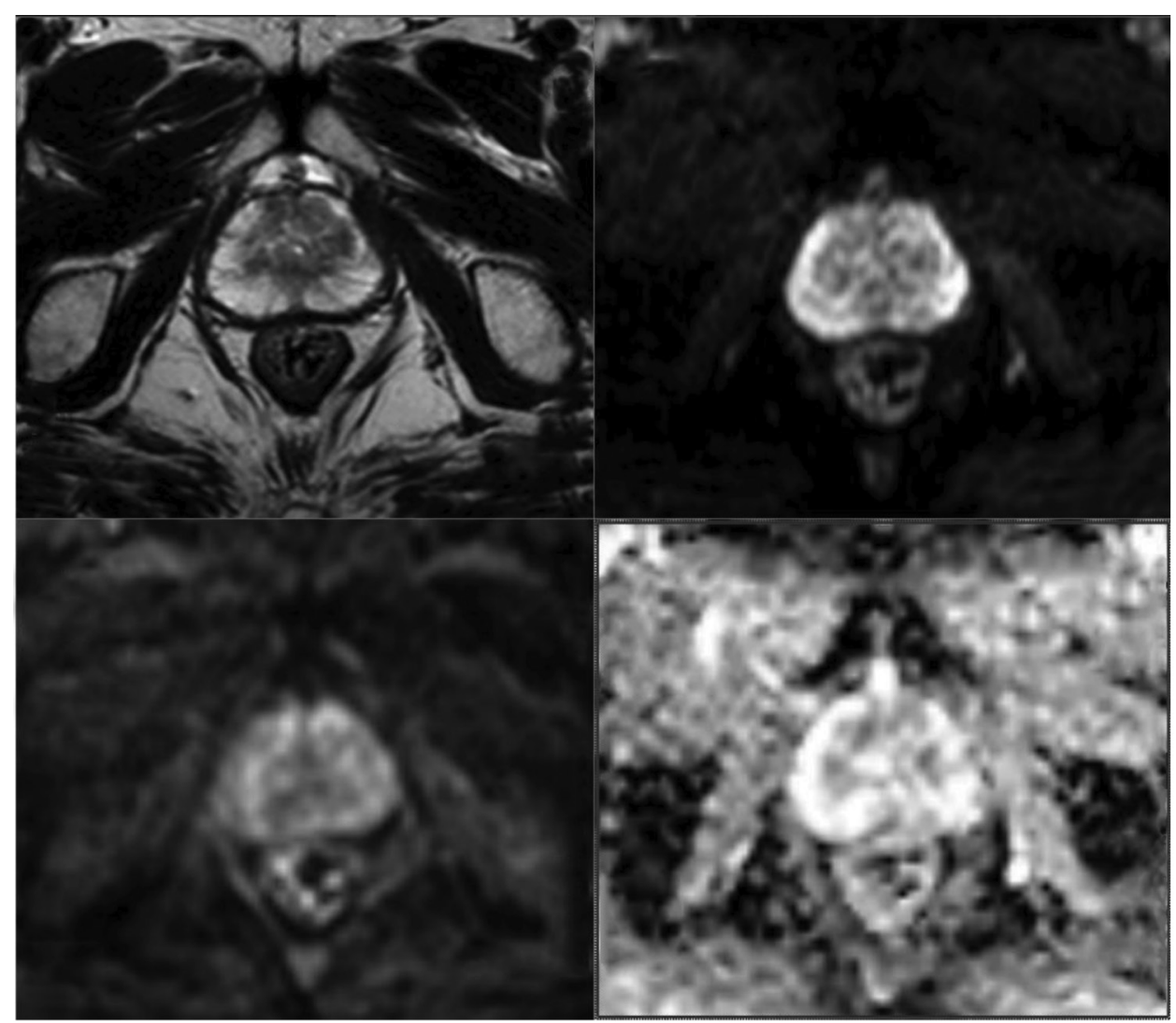

Fig. 2 Uniform high signal intensity T2 and low signal intensity on high b-values DWI and high signal on ADC-PIRADS 1.

and technical expertise, these lesions can either be biopsied or followed up (-Table 4).

Out of the aforementioned three sequences, T2W imaging and DWI are the mainstay for assessment. DCE plays the role of an adjunct ( - Fig. 7 ). Early enhancement within a lesion points toward a malignant lesion; however, it may be disregarded if a finding is a definite PIRADS 1 or 2 lesion based on the T2W images and DWI. Similarly, lack of early enhancement can be disregarded in lesions that are PIRADS 4 or 5 based on T2W images and DWI. However, DCE can tilt the scales in PIRADS 3 lesions where it assumes the role of a referee. For example, in a PIRADS 3 lesion in the PZ according to DWI, a positive DCE increases the likelihood of the finding being malignant, and the resultant finding can be upgraded to PIRADS category 4 (- Table 5).

In case the dominant sequence (DWI in the PZ and $\mathrm{T} 2$ in the TZ) is technically inadequate, PIRADS category " $\mathrm{X}$ " should be assigned and the sequence should be repeated. In case that is not possible, assessment can be made with other pulse sequences with a clear mention of the limitation in the report. 


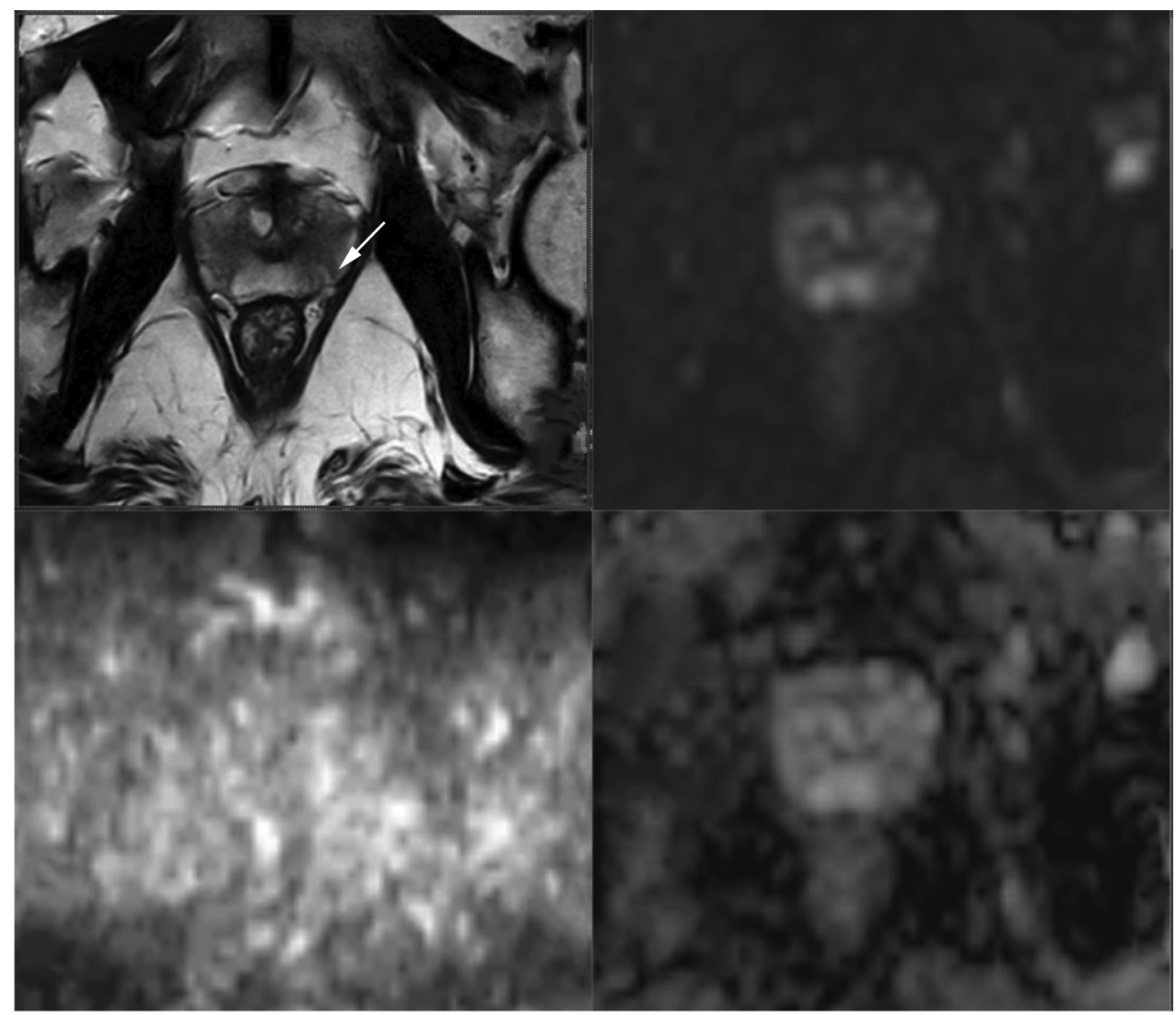

Fig. 3 Linear and wedge-shaped T2 hypointensities with indistinct margins without diffusion restriction-PIRADS 2.

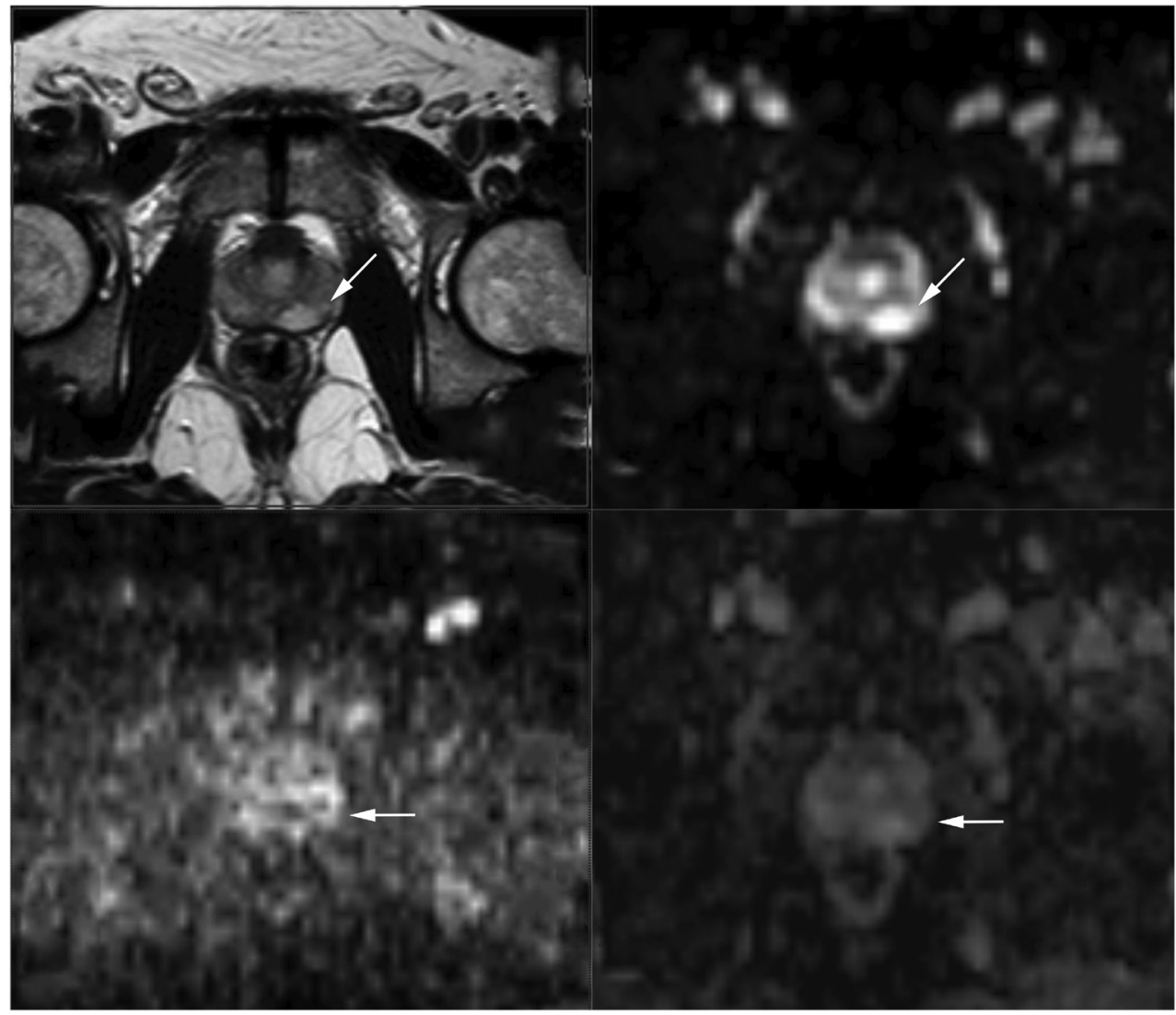

Fig. 4 T2 hypointense lesion with mild hyperintensity on high b-value DWI images with focal mild hypointensity on ADC in the left lateral peripheral zone (arrows)-PIRADS 3. 


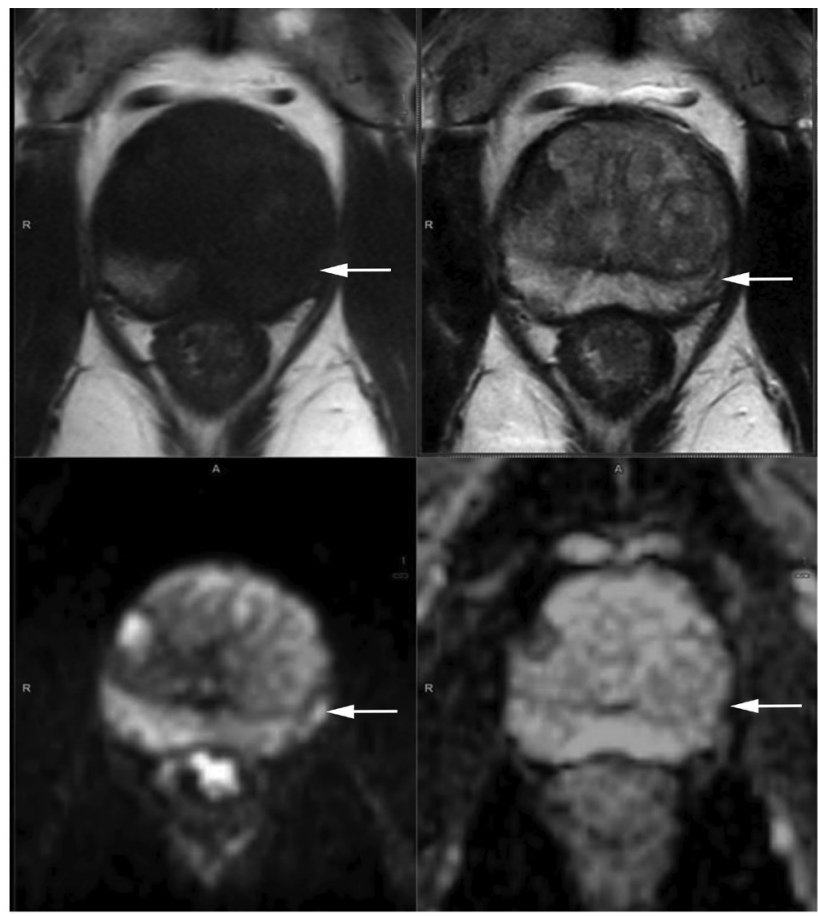

Fig. 5 Only an ill-defined T1 and T2 hypointensity in the left lateral peripheral zone. However, focal markedly hyperintense on high b-value DWI and hypointense area on ADC in left lateral peripheral zone measuring less than $1.5 \mathrm{~cm}$ (arrow)-PIRAD 4.

\section{Assessment without Adequate Dynamic Contrast} Enhancement

In PZ and TZ, it is determined by DWI assessment category (-Table 6). If both DWI and DCE are not available, only EPE should be assessed for the purpose of staging.

\section{Measurement of the Prostate Gland}

The volume of the prostate gland can either be calculated by automated segmentation or manually by measuring the maximum dimensions in all three planes using the following formula:

(Max anteroposterior diameter $) \times($ Max transverse diameter $) \times($ Max longitudinal diameter $) \times 0.52$.

This helps calculate the PSA density-PSA/prostatic volume.

\section{Mapping Lesions}

As prostatic malignancy can be multifocal, up to four findings with a PIRADS assessment category of 3, 4, or 5 may each be assigned on the sector map, and the index (dominant) intraprostatic lesion should be identified. The index lesion is the one which has the highest PIRADS assessment category. PIRADS v2 recommends that up to four findings with an assessment category of 3,4 , or 5 can be identified and reported. In case there are more than four lesions with suspicious findings, the four with the highest PIRADS assessment

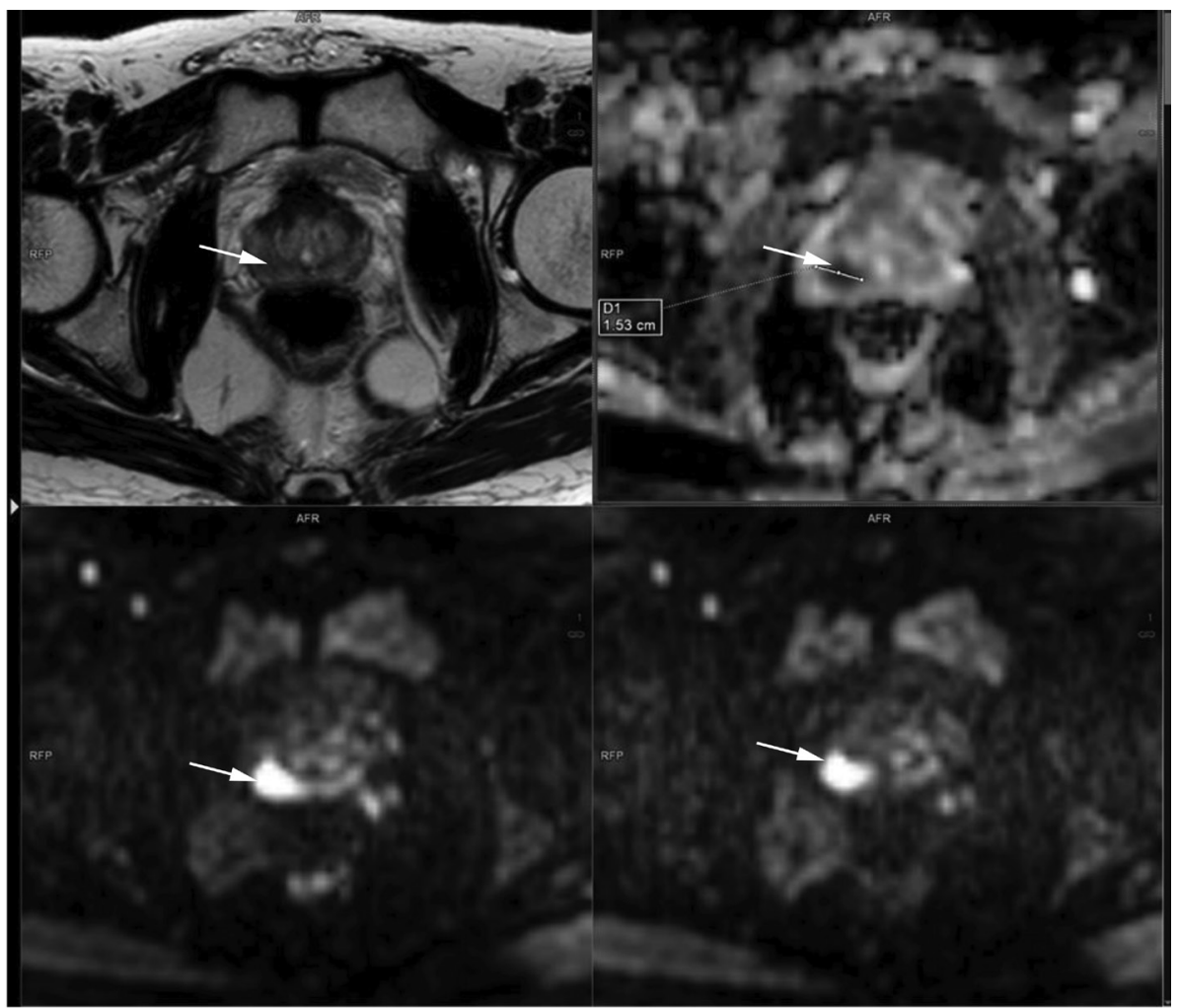

Fig. 6 A focal well-defined T2 hypointense region in the right peripheral zone, showing marked diffusion restriction appearing hypointense on ADC and hyperintense on high b value DWI with early enhancement on DCEI measuring more than $1.5 \mathrm{~cm}$. Features are consistent with a PIRADS 5 lesion. The lesion shows $>1 \mathrm{~cm}$ smooth contact with the adjacent capsule, a sign of EPE. Note the difference between PIRAD $4(<1.5 \mathrm{~cm})$ and PIRAD $5(>1.5 \mathrm{~cm})$ lesions are only size. Moreover, any capsular or NVB invading lesions are PIRAD 5 irrespective of size. 
Table 4 PIRADS v2 and the risk of malignancy

\begin{tabular}{|l|l|}
\hline PIRADS 1 & Very low (clinically significant cancer is highly unlikely to be present) \\
\hline PIRADS 2 & Low (clinically significant cancer is unlikely to be present) \\
\hline PIRADS 3 & Intermediate (the presence of clinically significant cancer is equivocal) \\
\hline PIRADS 4 & High (clinically significant cancer is likely to be present) \\
\hline PIRADS 5 & Very high (clinically significant cancer is highly likely to be present) \\
\hline
\end{tabular}

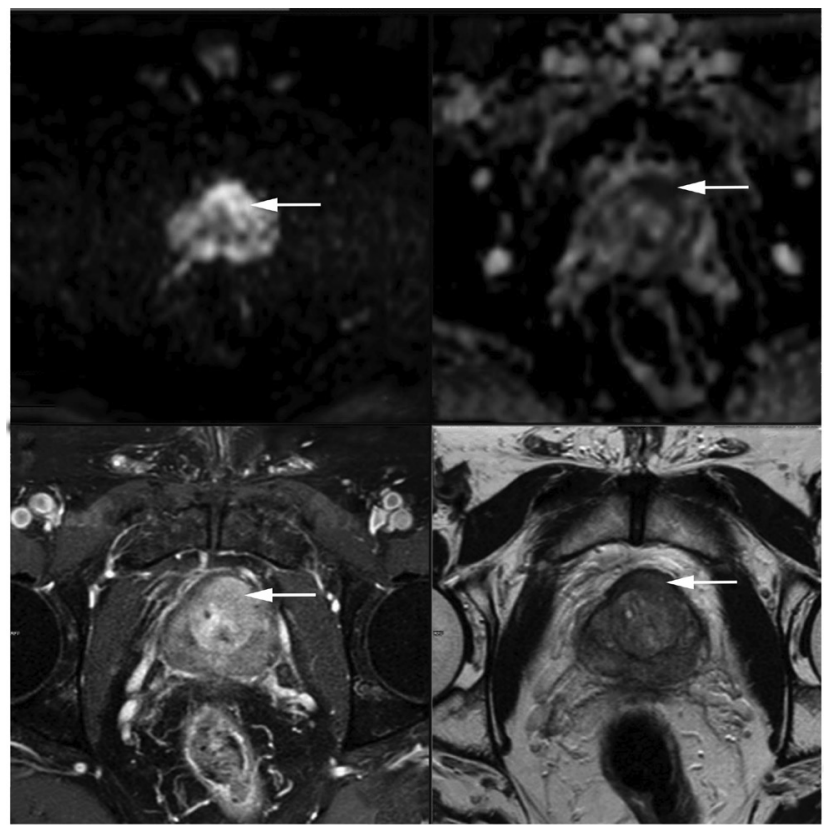

Fig. $7 \mathrm{~A}$ focal lesion in the left anterior fibromuscular stroma with extension into the adjacent central zone showing marked diffusion restriction appearing hyperintense on DWI, hypointense on ADC map showing early enhancement on DCEI and well-defined T2 hypointensity (arrows) s/o PIRAD 5 lesion highly suspicious of malignancy.

category should be reported. In case there are two lesions with the same PIRADS assessment category, the lesion that shows EPE should be the index lesion even if it is smaller in size. If neither lesions show EPE, then the larger lesion is considered the index lesion.

It is advised that benign findings such as cysts may be reported but is optional. They not only help as landmarks to guide subsequent biopsy or in follow-up but also help provide clarification to clinical colleagues who may see the lesion on films and have difficulty in interpretation. Ideally, the image number and series on which the measurement is made should be reported.

\section{Measurement of Lesions}

According to the PIRADS v2, the largest dimension of a suspicious finding should be reported with a mention of the plane (axial/sagittal/coronal) on which the measurement is made. Furthermore, for PZ lesions, the measurement should be made on ADC images, and for TZ lesions, measurement should be made on T2W images. However, in case lesion measurement is difficult on these sequences, it should be made on the sequence that best shows the lesion.
Table 5 PIRADS v2 final assignment of score

\begin{tabular}{|c|c|c|}
\hline \multicolumn{3}{|c|}{ Peripheral zone } \\
\hline DWI & DCE & Final PIRADS score \\
\hline 1 & Any & 1 \\
\hline 2 & Any & 2 \\
\hline \multirow[t]{2}{*}{3} & - & 3 \\
\hline & + & 4 \\
\hline 4 & Any & 4 \\
\hline 5 & Any & 5 \\
\hline \multicolumn{3}{|c|}{ Transition zone } \\
\hline $\mathrm{T} 2 \mathrm{~W}$ & DWI & Final PIRADS score \\
\hline 1 & Any & 1 \\
\hline 2 & Any & 2 \\
\hline \multirow[t]{2}{*}{3} & $\leq 4$ & 3 \\
\hline & 5 & 4 \\
\hline 4 & Any & 4 \\
\hline 5 & Any & 5 \\
\hline
\end{tabular}

Abbreviations: DCE, dynamic contrast enhancement; DWI, diffusion-weighted imaging; PIRADS, Prostate Imaging Reporting and Data System; T2W, T2-weighted.

Table 6 Assessment of PIRADS v2 without DWI

\begin{tabular}{|l|l|l|}
\hline \multicolumn{3}{|l|}{ Peripheral zone and transition zone } \\
\hline T2W & DCE & Final PIRADS score \\
\hline 1 & Any & 1 \\
\hline 2 & Any & 2 \\
\hline 3 & - & 3 \\
& + & 4 \\
\hline 4 & Any & 4 \\
\hline 5 & Any & 5 \\
\hline
\end{tabular}

Abbreviations: DCE, dynamic contrast enhancement; PIRADS, Prostate Imaging Reporting and Data System; T2W, T2-weighted.

\section{Staging}

To differentiate stage T2 EPE (tumor confined to the gland) from stage T3 EPE, MRI is a useful investigation. On MRI, it is essential to inspect the apex of the gland well. Invasion of the external urethral sphincter by cancer leads to a risk of surgically damaging the sphincter and results in urinary incompetence. Tumor in the apex of the gland may also need special considerations for radiation therapy. 
At times, obvious signs of EPE may be present, such as direct tumor extension into the bladder base or seminal vesicles with breach of the capsule. However, when there is no gross EPE, certain surrogate signs can predict EPE. The presence of a prostatic contour bulge or irregularity of prostatic margins, loss of normal rectoprostatic angle, asymmetry in the region of the NVBs, and a tumor-capsule contact length more than $1 \mathrm{~cm}$ can indicate EPE. Similarly, extension of malignancy into the seminal vesicle may be indicated by the features of seminal vesicle invasion including signal abnormality within the seminal vesicles in the form of $\mathrm{T} 2$ hypointensity, which may be focal or diffuse and/or abnormal contrast enhancement and/or restricted diffusion. Morphologically, loss of angle between the base of the prostate and the seminal vesicle may also be an indicator of seminal vesicle invasion.

The other important structures to be analyzed on MRI are the pelvic and retroperitoneal lymph nodes, that is, the common femoral, obturator, external iliac, internal iliac, common iliac, pararectal, presacral, and paracaval, and para-aortic lymph nodes. However, currently, the detection of abnormal lymph nodes is limited to size, morphology, and enhancement pattern. Although it is known that metastatic lymph nodes are not always enlarged, lymph nodes over $8 \mathrm{~mm}$ in size in the short axis are usually considered suspicious. One should also assess the images for the presence of skeletal metastases.

\section{Imaging Pearls}

- For proper correlation and synchronization between sequences, ensure that the imaging plane, location, and slice thickness for all sequences (T2W, DWI, and DCE) are the same.

- All signal abnormalities are not malignancies. In the PZ, signal alteration on T2W/DWI that is indistinct, linear, lobar, or diffuse, and not rounded may be secondary to prostatitis rather than malignancy.

- The ability of MRI to reliably detect and characterize malignancy in the PZ is more than that in the TZ.

- Homogeneous and heterogeneous nodules in the TZ that are round and well circumscribed are common in men above the age of 40 years. Irrespective of diffusion restriction and/or enhancement, they are considered to be benign BPH. They may sometimes harbor a malignancy but the probability is very low.

- Findings on DWI should always be correlated with those on T2W, T1W, and DCE imaging.

- Not all that appears dark on ADC images is malignant. Blood products, areas of fibrosis or dense fibromuscular stroma, and calcifications can be hypointense on T2 and ADC maps; however, they tend to be hypointense on high $b$-value images as well.
- $\mathrm{BPH}$ nodules are the most common benign finding that can masquerade as malignancy. Some of these nodules in the $\mathrm{TZ}$ are not clearly encapsulated and may show diffusion restriction. Also, some of these nodules can get extruded into the PZ and be well encapsulated and circumscribed with diffusion restriction. This is a limitation of mp-MRI.

- Signs of extracapsular extension:

- Asymmetric prostate capsular bulge with irregular margins.

- Obliteration of the rectoprostatic angle.

- Asymmetry of NVB.

- Tumor encasement of the NVB.

- Seminal vesicle invasion.

In conclusion, mp-MRI has emerged as an important tool for the detection and characterization of prostatic lesions. It now plays a quintessential role in the surveillance, diagnosis, and staging of PCa, as well as for the detection of local recurrence. As reliance on serum PSA has declined in the recent times, mp-MRI has emerged as the go-to tool for urologists all over the world.

\section{Conflict of Interest}

None declared.

\section{References}

1 Hariharan K, Padmanabha V. Demography and disease characteristics of prostate cancer in India. Indian J Urol 2016;32(2):103-108

2 Verma S, Rajesh A. A clinically relevant approach to imaging prostate cancer: review. Am J Roentgenol 2011;196(3 Suppl):S1-S10

3 Weinreb JC, Barentsz JO, Choyke PL, et al. PI-RADS Prostate Imaging-Reporting and Data System: 2015, version 2. Eur Urol 2016;69(1):16-40

4 Coakley FV, Hricak H. Radiologic anatomy of the prostate gland: a clinical approach. Radiol Clin North Am 2000;38(1):15-30

5 Allen KS, Kressel HY, Arger PH, Pollack HM. Age-related changes of the prostate: evaluation by MR imaging. Am J Roentgenol 1989;152(1):77-81

6 Hassanzadeh E, Glazer DI, Dunne RM, Fennessy FM, Harisinghani MG, Tempany CM. Prostate Imaging Reporting and Data System version 2 (PI-RADS v2): a pictorial review. Abdom Radiol (NY) 2017;42(1):278-289

7 Bonekamp D, Jacobs MA, El-Khouli R. Stoianovici D, Macura KJ. Advancements in MR imaging of the prostate: from diagnosis to interventions. Radiographics 2011;31(3):677-703

8 Barentsz JO, Richenberg J, Clements R, et al; European Society of Urogenital Radiology. ESUR prostate MR guidelines 2012. Eur Radiol 2012;22(4):746-757

9 Hedgire SS, Eberhardt SC, Borczuk R, McDermott S, Harisinghani MG. Interpretation and reporting multiparametric prostate MRI: a primer for residents and novices. Abdom Imaging 2014;39(5):1036-1051

10 Verma S, Turkbey B, Muradyan N, et al. Overview of dynamic contrast-enhanced MRI in prostate cancer diagnosis and management. Am J Roentgenol 2012;198(6):1277-1288 